\title{
A simple approach for fabricating solid-contact ion-selective electrodes using nanomaterials as transducers
}

\author{
Rongning Liang, Tanji Yin, Wei Qin* \\ Key Laboratory of Coastal Environmental Processes and Ecological Remediation, Yantai Institute of Coastal Zone Research (YIC), Chinese Academy of Sciences \\ (CAS), Shandong Provincial Key Laboratory of Coastal Environmental Processes, YICCAS, Yantai, Shandong 264003, PR China
}

\section{H I G H L I G H T S}

- A general method for fabricating nanomaterials based solid-contact ISEs is developed.

- The mixture of an ionic liquid and a nanomaterial is used as intermediate layer.

- The detection limits of the proposed sensors are in the nanomolar range.

- The developed electrodes exhibit a good response time and excellent stability.

\section{A R T I C L E I N F O}

\section{Article history:}

Received 3 August 2014

Received in revised form 14 October 2014

Accepted 24 October 2014

Available online 27 October 2014

\section{Keywords:}

Ion-selective electrodes

Solid contacts

Ion-to-electron transducers

Nanomaterials

Ionic liquids

\section{G R A P H I C A L A B S T R A C T}

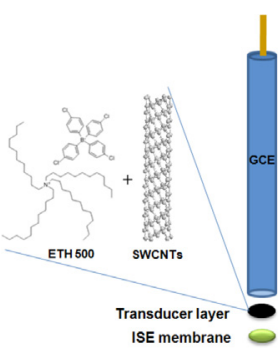

ISE membrane

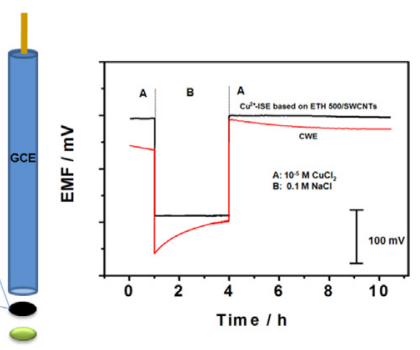

A B S T R A C T

A simple and robust approach for the development of solid-state ion-selective electrodes (ISEs) using nanomaterials as solid contacts is described. The electrodes are fabricated by using the mixture of an ionic liquid (IL) and a nanomaterial as intermediate layer, formed by melting the IL. Tetradodecylammonium tetrakis(4-chlorophenyl)borate (ETH 500) is chosen as an model of IL to provide strong adhesion between the inner glassy carbon electrode and the intermediate layer. Nanomaterials including single-walled carbon nanotubes (SWCNTs) and graphene were used as active ion-to-electron transducers between the glassy carbon electrode and the ionophore-doped ISE membrane. By using the proposed approach, the solid-contact $\mathrm{Cu}^{2+}$ - and $\mathrm{Pb}^{2+}$-selective electrodes based on ETH 500/SWCNTs and ETH 500/ graphene as transducers, respectively, have been fabricated. The proposed electrodes show detection limits in the nanomolar range and exhibit a good response time and excellent stability.

(c) 2014 Elsevier B.V. All rights reserved.

\section{Introduction}

Ion-selective electrodes (ISEs) are most widely used chemical sensors in clinical diagnostics, process control and environmental monitoring due to their intrinsic advantages including excellent selectivity, low cost, ease of use, and high reliability [1-3]. Among these, solid-contact ISEs which eliminate the internal solution and

\footnotetext{
* Corresponding author. Tel.: +86 535 2109156; fax: +86 5352109000 .

E-mail address: wqin@yic.ac.cn (W. Qin).
}

are easily miniaturized have been recognized as the means by which the next ISE generation will be constructed. Solid-contact ISEs, known as coated-wire electrodes (CWEs), were introduced many years ago [4]. However, such electrodes lack long-term stability and they are only useful in specific applications such as chromatographic detectors [5] or in flow-injection analysis [6]. Potential instabilities of CWEs have been mainly attributed to the lack of a well-defined redox couple and the formation of a water layer at the membrane-metal interface [7]. The historical problem of poor potential stability between the ion-selective membrane and the conducting wire has been largely solved by the 
introduction of conducting polymers [8] such as poly(3-octylthiophene) (POT) [9,10], polypyrrole (PPy) [11], polythiophene [12], polyaniline [13], and poly(3,4-ethylenedioxythiophene) [14] as ion-to-electron transducers. Although the ISEs based on these transducing materials show benefits compared to those of traditional ISE with an internal solution, they also suffer from some drawbacks such as sensitivity to light, $\mathrm{O}_{2}, \mathrm{CO}_{2}$, and $\mathrm{pH}[3]$.

In recent years, nanomaterials have been extensively studied and widely exploited in chemsensors and biosensors, since they possess some unique chemical, physical and electronic properties that cannot be achieved by their bulk counterparts [15]. Especially, the exceptional electrical properties such as the high charge transfer, the extraordinary electrical capacities and good hydrophobicity make them suitable as transducing components in potentiometric sensors [16,17]. Recently, many nanostructured carbon materials including fullerene [18], graphene [19,20], carbon nanotubes (CNTs) [21-23], three-dimensionally ordered macroporous (3DOM) carbon [24] and colloid-imprinted mesoporous (CIM) carbon [25] have been utilized as ion-to-electron transducers in solid-contact ISE. The electrodes based on these nanomaterials as transducers exhibit excellent response performances such as long-term potential stability, and insensitivity to $\mathrm{O}_{2}$. It has been well known that the transducer layer of solid-contact ISEs based on nanomaterials is usually formed by drop casting a suspension solution of nanomaterial onto the surface of the inner electrode (e.g., glassy carbon electrode, GCE) which is followed by heating the electrode surface to evaporate the solvent with a hot air stream or an infrared lamp. In order to obtain a homogeneous coverage of nanomaterials on the surface of the electrode, these processes have to be repeated for many times [18-22]. It is clear that such fabrication processes are somewhat complicated and time consuming. In addition, nanomaterials can easily peel off from the electrode surface owing to the poor adhesion between these nanomaterials and the surface of the GCE. This poses serious limits to their use in solid-contact ISEs.

Ionic liquids (ILs) refer to liquids composed entirely of ions that are fluid around or below $100^{\circ} \mathrm{C}$. They possess unique features, such as low volatility, tunable viscosity, high conductivity, large electrochemical window and low toxicity. These properties make ILs particularly suitable for their use in electrochemical sensors [26-29].

Herein, we propose a simple and robust approach for fabricating solid-contact ISEs based on nanomaterials as transducers. The transducer layer can be prepared by simply spreading the IL and nanomaterial composite on the GCE. Tetradodecylammonium tetrakis(4-chlorophenyl) borate (ETH 500) with a melting point of $79^{\circ} \mathrm{C}$, is a kind of IL but not a room-temperature IL. ETH 500 is utilized to provide strong adhesion between the GCE and the nanomaterial layer. In this work, single-walled carbon nanotubes (SWCNTs) and graphene have been chosen as the models of nanomaterials since they have been extensively used as solid contacts in potentiometric sensors. The chemical structure of ETH 500 and schematic structures of SWCNTs and graphene are shown in Scheme 1. By using the proposed approach, solid-contact $\mathrm{Cu}^{2+}$ and $\mathrm{Pb}^{2+}$-selective electrodes based on ETH 500/SWCNTs and ETH 500/ graphene as ion-to-electron transducers, respectively, have been fabricated. It shows that the proposed approach offers a simple way for fabrication of reliable and high-performance solid-contact ISEs based on nanomaterials as ion-to-electron transducers.

\section{Experimental}

\subsection{Reagents}

The ionophores $N, N, N^{\prime}, N^{\prime}$-tetradodecyl-3,6-dioxaoctanedithioamide (ETH 1062, $\mathrm{Cu}^{2+}$ ionophore), tert-butylcalix[4]arene-tetrakis( $N, N$-dimethylthioacetamide) (lead ionophore IV), tetradodecylammonium tetrakis(4-chlorophenyl) borate (ETH 500 ), high molecular weight poly (vinyl chloride) (PVC), 2nitrophenyl octyl ether (o-NPOE), bis(2-ethylhexyl) sebacate (DOS), sodium tetrakis[3,5-bis(trifluoromethyl)phenyl]borate (NaTFPB), and dimethylformamide (DMF) were purchased from Fluka AG (Buchs, Switzerland). SWCNTs and graphene were obtained from XFnano Materials Tech Co., Ltd. (Nanjing, China). Aqueous solutions were prepared with freshly deionized water (18.2 $\mathrm{M} \Omega \mathrm{cm}$ specific resistance) obtained with a Pall Cascada laboratory water system. Tetrahydrofuran (THF) was freshly distilled prior to use. All other reagents were analytical grade and used without any further purification. A stock solution of $0.01 \mathrm{M} \mathrm{Cu}^{2+}$ or $\mathrm{Pb}^{2+}$ was prepared by dissolving $\mathrm{CuCl}_{2}$ or $\mathrm{Pb}\left(\mathrm{NO}_{3}\right)_{2}$ in deionized water and then diluted to various concentrations of working solutions with deionized water prior to measurements.

\subsection{Apparatus}

All measurements of electromotive force (EMF) were performed at $20-21^{\circ} \mathrm{C}$ using a PXSJ-216 pH meter (Leici, Shanghai) in the galvanic cell: saturated calomel electrode (SCE)//0.1 M LiOAc/ sample solution/ISE membrane/nanomaterial layer/GCE. The external reference electrode employed was a double-junction

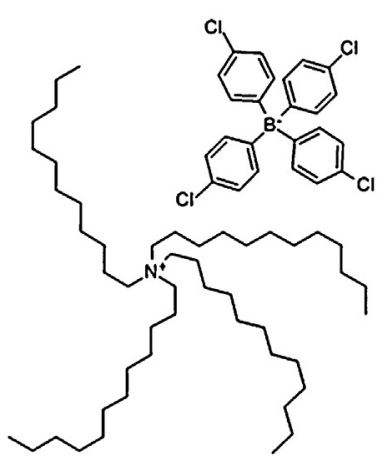

ETH 500

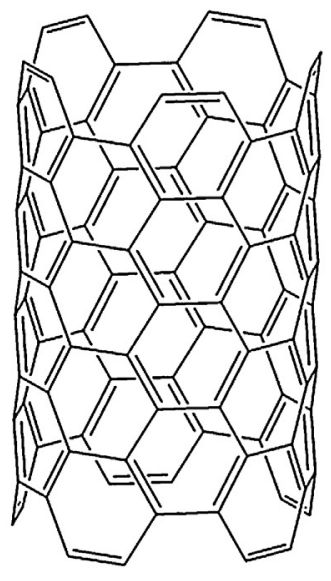

SWCNTs

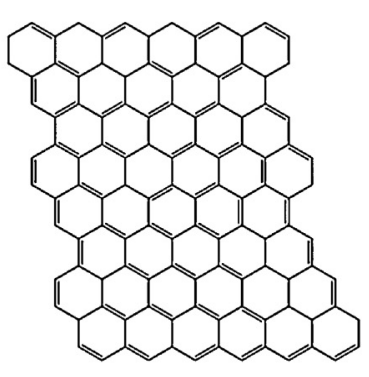

graphene

Scheme 1. Chemical structure of ETH 500 and the schematic structures of SWCNTs and graphene. 
SCE with $0.1 \mathrm{M}$ LiOAc as a bridge electrolyte. Selectivity coefficients were determined by the separate solution method [30]. The EMF values were corrected for the liquid-junction potentials with the Henderson equation. The activity coefficients of ions were calculated from the modified Debye-Hückel equation [31].

Electrochemical impedance spectroscopy (EIS) measurements were performed by using a $\mathrm{CHI} 760 \mathrm{C}$ electrochemical workstation (Shanghai Chenhua Instruments, China) with a three-electrode configuration. The reference electrode was an $\mathrm{Ag} / \mathrm{AgCl}(3 \mathrm{M} \mathrm{KCl})$, and the auxiliary electrode was a platinum wire. The impedance spectra were recorded at open-circuit potential in deaerated $0.1 \mathrm{M} \mathrm{CuCl}_{2}$ solution with the excitation amplitude of $10 \mathrm{mV}$ and a frequency range of $100 \mathrm{kHz}-0.3 \mathrm{~Hz}$ by using a sinusoidal excitation signal.

\subsection{Preparation procedures}

Different membrane cocktails were prepared by dissolving $360 \mathrm{mg}$ of components in $2.5 \mathrm{~mL}$ of THF:ETH 1062 ( $1 \mathrm{wt} \%$, $11.3 \mathrm{mmol} \mathrm{kg}{ }^{-1}$ ), NaTFPB ( $1 \mathrm{wt} \%, 10.9 \mathrm{mmol} \mathrm{kg}^{-1}$ ), o-NPOE (49 wt $\%)$, and PVC $(49 \mathrm{wt} \%)$ for the $\mathrm{Cu}^{2+}$-selective electrode; lead ionophore IV ( $1 \mathrm{wt} \%, 9.5 \mathrm{mmol} \mathrm{kg}{ }^{-1}$ ), NaTFPB (0.5 wt\%, $5.4 \mathrm{mmol}$ $\mathrm{kg}^{-1}$ ), ETH 500 ( $1 \mathrm{wt} \%, 8.7 \mathrm{mmol} \mathrm{kg}^{-1}$ ), DOS (49wt\%), and PVC (48.5 wt\%) for the $\mathrm{Pb}^{2+}$-selective electrode. The membrane cocktails were degassed by sonication for $10 \mathrm{~min}$.

The fabrication procedure for the solid-contact ISEs based on ETH 500/nanomaterials was described as follows: (1) GCE was polished with $0.3 \mu \mathrm{m}$ alumina slurries and rinsed with water, then dried with acetone, and left in $\mathrm{CH}_{3} \mathrm{Cl}_{3}$ for $10 \mathrm{~min}$. Finally, the resulting GCE was inserted into a piece of matched PVC tubing at the distal end. (2) $15 \mathrm{mg}$ of ETH 500 and $1 \mathrm{mg}$ of SWCNTs or graphene were fully mixed. The mixture was spread onto the electrode surface and heated by an infrared lamp placed $5 \mathrm{~cm}$ above the electrode for $10 \mathrm{~s}$ to melt ETH 500 and then left to cool. A uniform composite layer with strong adhesion to the GCE surface was obtained. (3) $50 \mu \mathrm{L}$ of the membrane cocktail was drop-cast onto the transducer layer and allowed to dry for $2 \mathrm{~h}$. The CWEs were prepared by the similar procedure except for omission of solid contacts.

The conventional solid-contact electrodes using nanomaterials as transducers were fabricated according to Rius's method with some modifications [21]. Briefly, $0.5 \mathrm{mg}$ of nanomaterials were dispersed in $10 \mathrm{~mL}$ DMF by sonication for $4 \mathrm{~h}$, and then $40 \mu \mathrm{L}$ of nanomaterials/DMF solution was drop-cast onto the polished GCE surface and dried by an infrared lamp. These processes were performed in 20 rounds to obtain a homogeneous coverage of nanomaterials on the surface of the electrode. Finally, $50 \mu \mathrm{L}$ of the membrane cocktail was drop-cast onto the transducer layer and allowed to dry for $2 \mathrm{~h}$.

The $\mathrm{Cu}^{2+}$-selective electrodes were firstly conditioned in $10^{-3} \mathrm{M}$ $\mathrm{CuCl}_{2}$ for 1 day and then in $10^{-9} \mathrm{M} \mathrm{CuCl}_{2}$ for 2 days. The $\mathrm{Pb}^{2}$ ${ }^{+}$-selective electrodes were conditioned first in $10^{-3} \mathrm{M} \mathrm{Pb}\left(\mathrm{NO}_{3}\right)_{2}$ for 1 day and subsequently in $10^{-9} \mathrm{M} \mathrm{Pb}\left(\mathrm{NO}_{3}\right)_{2}$ for another day.

\section{Results and discussion}

\subsection{Response performance of the solid-contact $\mathrm{Cu}^{2+}$-ISE based on ETH 500/SWCNTS}

We employed here a simple method for fabrication of robust solid-contact ISEs using SWCNTs as transducer. The transducer layer was prepared by simply spreading ETH 500 and SWCNTs mixture in the presence of a heat source onto the GCE surface and melting ETH 500. After cooling, a robust transducer layer was achieved. The micrographs of the mixture of ETH 500 and SWCNTs before heating and after cooling were characterized by scanning electron micrography (SEM). The SEM images are shown in Fig. S1 in the Supporting information. As shown, a solid powder mixture is obtained before heating, while a uniform layer is formed on the electrode surface after cooling. The solid-contact $\mathrm{Cu}^{2+}$-ISE based on this method was constructed as model system. The potential response associated with the proposed $\mathrm{Cu}^{2+}$-selective sensor is shown in Fig. 1. Clearly, the electrode based on ETH 500/SWCNTs exhibits excellent response performance. The calibration plot was recorded over a range between $1.0 \times 10^{-4}$ and $1.0 \times 10^{-10} \mathrm{M}$. The proposed electrode shows a Nernstian response of $29.8 \mathrm{mV} /$ decade over a wide concentration range of $1.0 \times 10^{-4}$ to $1.0 \times 10^{-8} \mathrm{M}$ with a detection limit of $4.0 \times 10^{-9} \mathrm{M}$ (calculated as the intersection of the two slopes). In addition, it can be also seen that the proposed electrode functions equivalently, in terms of the copper response, to the conventional SWCNTs based ISE with a detection limit of $4.0 \times 10^{-9} \mathrm{M}$ (Fig. 1b). Thus, it can be demonstrated that the introduction of the ion-to-electron transducer based on ETH 500/ SWCNTs does not affect the potential response performance of the solid-contact ISE. Additionally, the ETH 500/SWCNTs based solidcontact electrode exhibited a fast response time of $\leq 10 \mathrm{~s}$. It can be deduced that the elimination of an inner solution favors the response time of the solid-contact electrode [32,33].

The selectivity coefficients of the ETH 500/SWCNTs based $\mathrm{Cu}^{2}$ +-ISE for interfering ions were determined by the separate solution method to eliminate the influence of the inherent sensitivity limit on the ISE response toward discriminated ions [30]. The results are summarized in Table 1 . It can be seen that the potentiometric selectivity coefficients of the proposed solid-contact electrode are comparable to those of the conventional liquid-contact electrode based on the same ionophore [34].

\subsection{Water film test and long-term stability}

Potentiometric water film test was performed to evaluate the formation of an aqueous layer between the $\mathrm{Cu}^{2+}$-selective membrane and the ETH 500/SWCNTs layer. To test for the presence of a water layer, the ISEs were first conditioned in a solution of the primary ions and then the sample was replaced with a solution of the interfering ions [35]. A control experiment was performed by using the CWE. The results for the water film test are shown in Fig. 2. As can be seen, a negative EMF change of ca. $180 \mathrm{mV}$ is observed for both electrodes upon replacing the $\mathrm{CuCl}_{2}$ solution with the $\mathrm{NaCl}$ solution. Such EMF change reflects the change in the

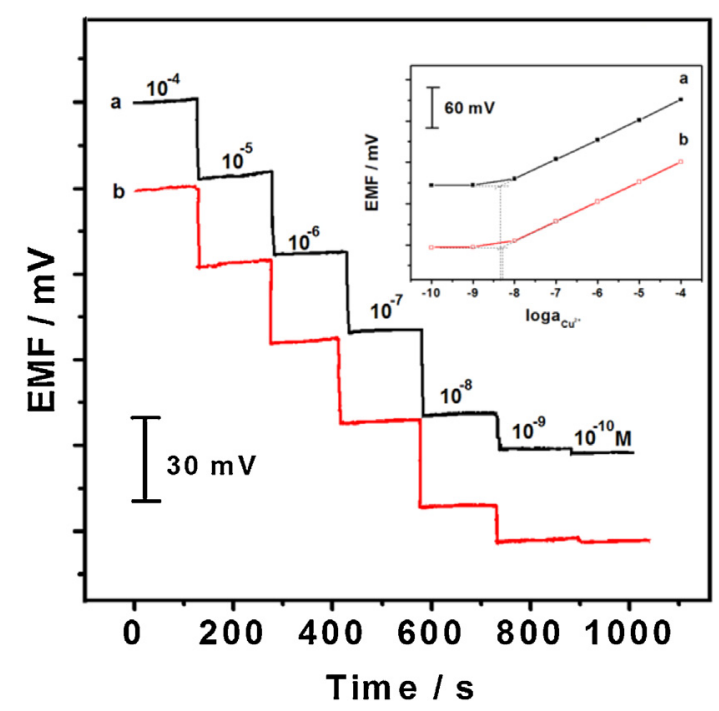

Fig. 1. Recorded potential time traces for the proposed ETH 500/SWCNTs based $\mathrm{Cu}^{2}$ ${ }^{+}-$ISE (a) and the conventional SWCNTs based $\mathrm{Cu}^{2+}$-ISE (b). The inset shows the corresponding calibration curves. 
Table 1

Potentiometric selectivity coefficients, $\log K_{\mathrm{Cu} . \mathrm{J}}^{\text {pot }}$ of the proposed solid-contact $\mathrm{Cu}^{2}$ ${ }^{+}$-ISE compared with those obtained with the conventional liquid-contact ISE [34].

\begin{tabular}{llc}
\hline Ion J & \multicolumn{1}{l}{$\log K_{\text {Cu.J }}^{\text {pot a }}$} & \\
\cline { 2 - 3 } & Solid-contact ISE & Liquid-contact ISE \\
\hline $\mathrm{Na}^{+}$ & $-10.5 \pm 0.3$ & $-10.8 \pm 0.1$ \\
$\mathrm{~K}^{+}$ & $-8.6 \pm 0.1$ & $-8.9 \pm 0.2$ \\
$\mathrm{Ca}^{2+}$ & $-11.9 \pm 0.4$ & $-11.4 \pm 0.1$ \\
$\mathrm{Mg}^{2+}$ & $-13.3 \pm 0.6$ & $-13.9 \pm 0.5$ \\
\hline
\end{tabular}

a Mean value obtained from three corresponding pairs of concentrations of $\mathrm{Cu}^{2+}$ and the respective interfering cation in the Nernstian response range \pm SD.

outer phase boundary potential as a consequence of the selectivity behavior of the $\mathrm{Cu}^{2+}$-selective membrane [21]. In this process, an obvious positive potential drift is observed for the CWE, while the electrode based on ETH 500/SWCNTs exhibits a stable potential response. It has been well known that the positive drifts are indicative of the presence of a water layer between the membrane and the solid contact [7]. Thus, it can be demonstrated that the undesirable water layer could be successfully eliminated by the intermediate layer of the proposed electrode. The high hydrophobicity of the ETH 500/SWCNTs based transducer layer may lead to the absence of the water film.

The potential stability of the proposed solid-contact $\mathrm{Cu}^{2+}$-ISEs was tested. The intermediate-term stability was calculated with the signal recorded in the third step of the water layer test. The obtained potential drift for the ETH 500/SWCNTs based $\mathrm{Cu}^{2+}$-ISE is $352 \mu \mathrm{Vh}^{-1}$ for the $6 \mathrm{~h}$ continuous monitoring, which is much lower than that of the CWE $\left(3125 \mu \mathrm{Vh}^{-1}\right)$. The long-term stability of the solid-contact $\mathrm{Cu}^{2+}$-ISE based on ETH 500/SWCNTs was investigated by measuring the responses to copper ions from $1.0 \times 10^{-4}$ to $1.0 \times 10^{-10} \mathrm{M}$ with the same electrode on different days. Before measurements, the electrode was stored in $1.0 \times 10^{-9} \mathrm{M} \mathrm{CuCl}_{2}$ solution. The results are shown in Fig. 3. No significant loss of the electrode slope $\left(28.6 \mathrm{mVdecade}^{-1}\right)$ is observed after continuous use for two weeks. Note that the $\mathrm{E}^{\circ}$ values of the proposed electrode were found to be changeable during this period, probably due to the generation of the reduced and oxidized species (e.g., $\mathrm{O}_{2}$ ) and the water absorption of the solvent polymeric membrane [36]. The detection limit increased by $c a$. half an order of magnitude after 3 weeks. This long-term stability of the proposed sensor might be due to the fact that the high lipophilicity of SWCNTs, and the physically robust nature and good adhesion properties of ETH 500 prohibit the formation of the

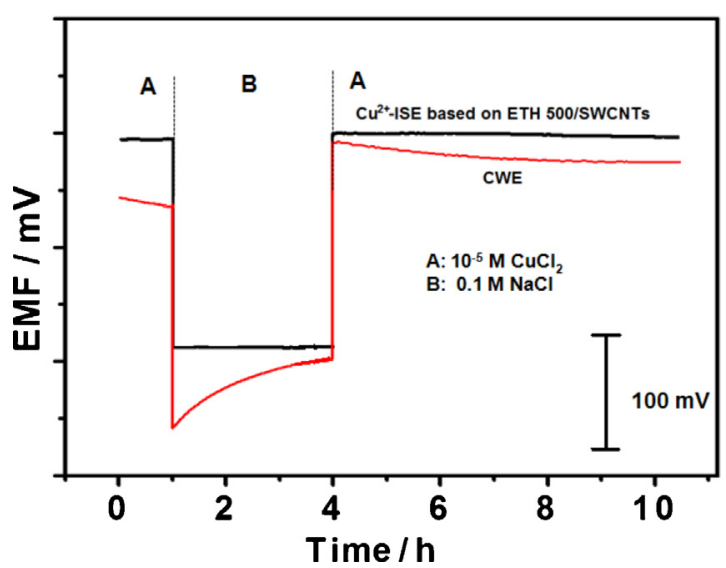

Fig. 2. Water film tests of the solid-contact $\mathrm{Cu}^{2+}$-ISE based on ETH 500/SWCNTS and of the CWE. At $t=1 \mathrm{~h}$, the solution of the primary ion $\left(10^{-5} \mathrm{M} \mathrm{CuCl}_{2}\right)$ was exchanged to $10^{-1} \mathrm{M} \mathrm{NaCl}$. At $t=4 \mathrm{~h}$, the sample was replaced by the initial solution.

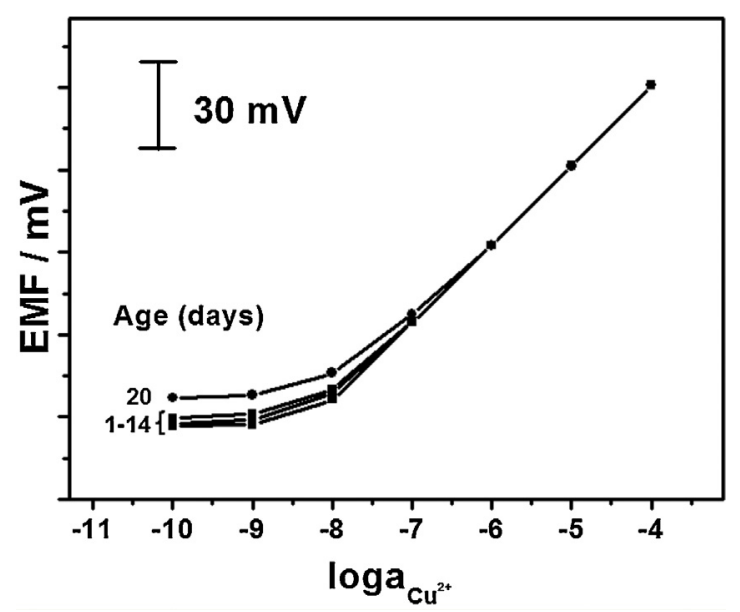

Fig. 3. Long-term stability of the proposed solid-contact $\mathrm{Cu}^{2+}$-ISE.

detrimental water layer. These results suggest that the proposed solid-contact ISEs exhibit an excellent potential stability.

In addition, the proposed electrode was found to show a good reproducibility. For $100 \mathrm{nM} \mathrm{Cu}{ }^{2+}$, the relative standard deviation (RSD\%) of six different electrodes was found to be 1.6\%. Furthermore, the influence of $\mathrm{O}_{2}$ on the potential stability of the proposed solid contact electrode was investigated in a stirred solution of $10^{-3} \mathrm{M}$ $\mathrm{CuCl}_{2}$ by first purging with $\mathrm{N}_{2}$ for $0.5 \mathrm{~h}$, then $\mathrm{O}_{2}$ for $0.5 \mathrm{~h}$ and last $\mathrm{N}_{2}$ for $0.5 \mathrm{~h}$. The results are shown in Fig. S2 in the Supporting information. As can be seen, the electrode based on ETH 500/SWCNTs as the solidcontact layer is insensitive to $\mathrm{O}_{2}$, while the CWE shows a potential drift of over $20 \mathrm{mV}$ when $\mathrm{O}_{2}$ is introduced into the sample. This further confirms the inherent advantages of ETH 500/SWCNTs acting as the solid-contact transducer.

\subsection{Characterization by EIS}

The EIS measurements were applied to characterize the proposed electrode. Fig. S3 (see Supporting information) shows the impedance plots of the GCE/ETH 500/SWCNTs recorded in 0.1, $0.05,0.01 \mathrm{M} \mathrm{CuCl}_{2}$ solutions. All the impedance spectra are dominated by a near $90^{\circ}$ capacitive line. A slight deviation from the capacitive line is found at the high frequencies, indicating the fast charge transfer at the GCE/ETH 500/SWCNTs and ETH 500/ SWCNTs/solution interfaces as well as the ETH 500/SWCNTs layer [32]. Additionally, the values of high-frequency intersection with the $Z^{\prime}$ axis depend highly on the electrolyte concentration, indicating that the measured impedance is primarily the solution resistance but not the resistance of the ETH 500/SWCNTs layer. It can be also seen that the values of low-frequency impedance $\left(-Z^{\prime \prime}\right)$ increase with decreasing the concentrations of the electrolyte. According to the equation $C=-1 /\left(2 \pi f Z^{\prime \prime}\right)$ (where $f$ is the frequency and $Z^{\prime \prime}$ is the imaginary part of the impedance), it can be deduced that the bulk capacitance decreases with decreasing the electrolyte concentration, which is consistent with the previous report [37].

The EIS response of all systems was modeled using ZSimpWin Version 3.10 software, and an equivalent circuit (see Fig. S4 in the Supporting information) was employed in the circuit modeling. The values of the respective resistances, capacitances and $n$-factors are summarized in Table S1 in the Supporting information. The impedance behaviors of ETH 500/SWCNTs based $\mathrm{Cu}^{2+}$-ISE and the CWE are compared in Fig. 4. As shown in Table S1 and Fig. 4, the resistance, which is equal to the bulk membrane resistance coupled with the contact resistance between the electrode substrate and the ISE membrane, decreases from 0.74 to $0.49 \mathrm{M} \Omega$ with the presence of the ETH 500/SWCNTs layer which facilitates the charge transport at 


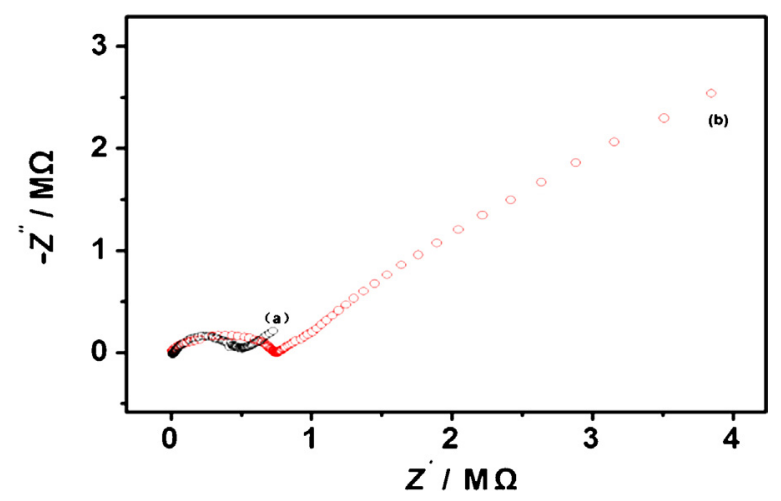

Fig. 4. EIS data for the the proposed $\mathrm{Cu}^{2+}$-ISE based on ETH 500/SWCNTs (a) and for the CWE (b).

the interface. At the low frequencies, a large semicircle is found at the EIS of the CWE, indicating a large charge-transfer resistance with a small capacitance at the "blocked" interface of the CWE. The negligible of low-frequency semicircle in the case of the solidcontact ISE shows that the ETH 500/SWCNTs contact increases the low-frequency capacitance and facilitates the ion-to-electron transduction between the electrode substrate and the ion-selective membrane [38].

\subsection{Further application}

The satisfactory results obtained for $\mathrm{Cu}^{2+}$-ISE motivated us to test similar systems using other nanomaterials as solid contacts in order to develop a general and simple strategy for fabrication of robust solid-contact electrodes based on nanomaterials. As a further example, solid-contact $\mathrm{Pb}^{2+}$-selective electrode based on ETH 500/graphene as ion-to-electron transducer was fabricated. The time-dependent EMF response traces and calibration curves of the $\mathrm{Pb}^{2+}$-selective electrodes are shown in Fig. 5. It can be seen that the lead electrode based on the proposed transducer (ETH 500 /graphene) shows a $1.8 \times 10^{-9} \mathrm{M}$ detection limit and Nernstian response slopes as well, which is essentially identical to the conventional solid-contact $\mathrm{Pb}^{2+}$-selective electrode using graphene as transducer which exhibits a $1.7 \times 10^{-9} \mathrm{M}$ detection limit. This good correspondence demonstrates the robustness of the approach. The corresponding response times for repeated alternate measurements of $1.0 \times 10^{-4}$ and $1.0 \times 10^{-9} \mathrm{M} \mathrm{Pb}^{2+}$ were

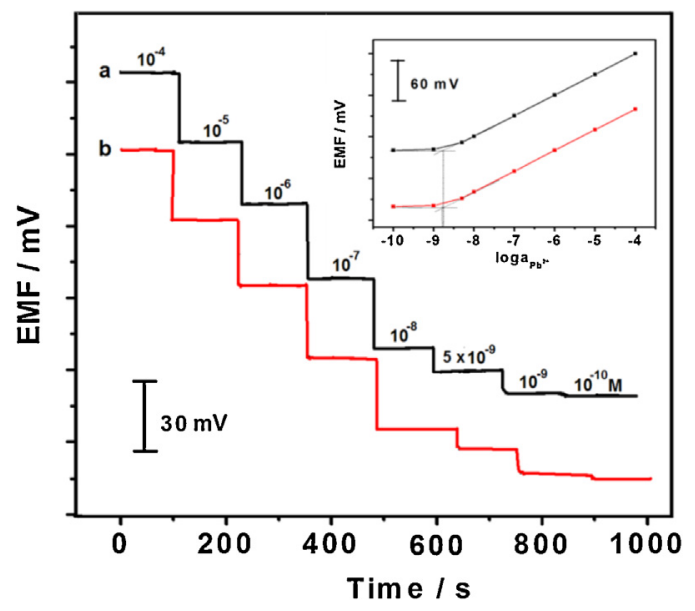

Fig. 5. Time responses obtained with the solid-contact $\mathrm{Pb}^{2+}$-ISE based on ETH 500/ graphene (a) and the conventional solid-contact $\mathrm{Pb}^{2+}$-ISE based on graphene (b). Inset: corresponding potentiometric response curves.

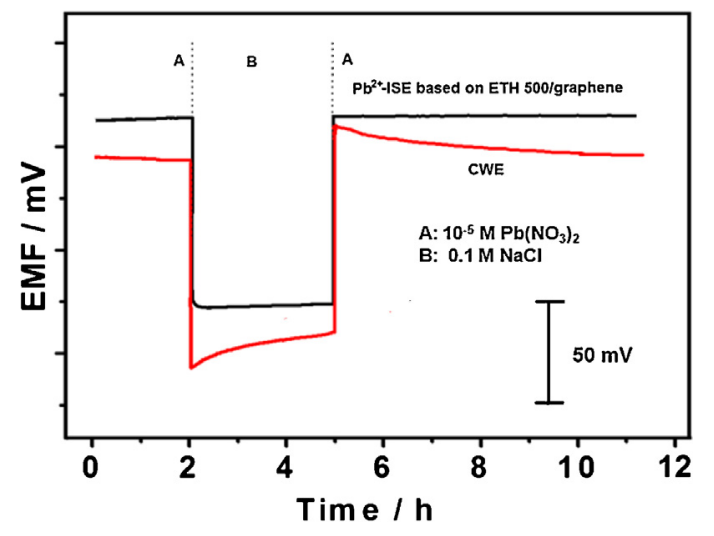

Fig. 6. Response of the solid-contact $\mathrm{Pb}^{2+}$-ISE based on ETH 500/graphene as transducer layer (a) and the CWE (b). At $t=2 \mathrm{~h}$ the solution of the primary ion $\left(10^{-5} \mathrm{M} \mathrm{Pb}\left(\mathrm{NO}_{3}\right)_{2}\right)$ was replaced with $10^{-1} \mathrm{M} \mathrm{NaCl}$, which, in turn, was replaced with the initial solution at $t=5 \mathrm{~h}$.

$<10$ and $<20 \mathrm{~s}$ for the higher and lower concentrations, respectively.

The water layer test of the solid-contact $\mathrm{Pb}^{2+}$-selective electrode was also performed. Fig. 6 exhibits the potential response of the proposed $\mathrm{Pb}^{2+}$-ISE based on ETH 500/graphene and the $\mathrm{CWE}$ measured in $10^{-5} \mathrm{M} \mathrm{Pb}\left(\mathrm{NO}_{3}\right)_{2}, 10^{-1} \mathrm{M} \mathrm{NaCl}$ and again $10^{-5} \mathrm{M} \mathrm{Pb}\left(\mathrm{NO}_{3}\right)_{2}$. It is clear that an obvious potential drift is observed for the CWE when changing back from $\mathrm{Na}^{+}$to $\mathrm{Pb}^{2+}$. This indicates that a water film was formed between the GCE and the solid contact. However, no such drift was found when using the ETH 500/graphene as the solid-contact layer. This demonstrates that the water layer was also eliminated by the highly hydrophobic ETH 500/graphene layer. This result further confirms the utility of the proposed approach.

\section{Conclusions}

In this work, a simple and robust approach for fabrication of the solid-contact ISE using nanomaterials has been proposed. The method is based on the combination of the excellent properties of the nanomaterials and the good adhesion ability of the ionic liquid of ETH 500. Compared to the CWEs, the proposed solid-contact ISEs based on ETH 500/SWCNTs and ETH 500/graphene as transducers exhibit significantly improved potential stability. In addition, the electrodes demonstrate excellent sensing properties including a wide dynamic response range, fast response time and long-term stability. The proposed approach can be extended to the development of solid-contact sensors based on other nanomaterials. It is anticipated that such a simplified preparation procedure will make it possible for other researchers to more easily develop and apply potentiometric sensors based on nanomaterials.

\section{Acknowledgements}

This work was financially supported by the Instrument Developing Project of the Chinese Academy of Sciences (YZ201161), The National Natural Science Foundation of China (41206087,21475148) and The National 863 High Technology Project of the Ministry of Science and Technology of China (2007AA09Z103). Many thanks to Mr. Kunhao Yang and Mr. Heng Cao for preliminary experiments.

\section{Appendix A. Supplementary data}

Supplementary data associated with this article can be found, in the online version, at http://dx.doi.org/10.1016/j.aca.2014.10.033. 


\section{References}

[1] M. Pawlak, E. Bakker, Chemical modification of polymer ion-selective membrane electrode surfaces, Electroanalysis 26 (2014) 1121-1131.

[2] A. Bratov, N. Abramova, A. Ipatov, Recent trends in potentiometric sensor arrays - a review, Anal. Chim. Acta 678 (2010) 149-159.

[3] J. Bobacka, A. Ivaska, A. Lewenstam, Potentiometric ion sensors, Chem. Rev. 108 (2008) 329-351.

[4] R.W. Cattrall, H. Freiser, Coated wire ion-selective electrodes, Anal. Chem. 43 (1971) 1905-1906.

[5] P. Schnierle, T. Kappes, P.C. Hauser, Capillary electrophoretic determination of different classes of organic ions by potentiometric detection with coated-wire ion-selective electrodes, Anal. Chem. 70 (1998) 3585-3589.

[6] T. Dimitrakopoulos, J.R. Farrell, P.J. Iles, A photo-cured calcium ion-selective electrode for use in flow injection potentiometry that tolerates high perchlorate levels, Electroanalysis 8 (1996) 391-395.

[7] M. Fibbioli, W.E. Morf, M. Badertscher, N.F. de Rooij, E. Pretsch, Potential drifts of solid-contacted ion-selective electrodes due to zero-current ion fluxes through the sensor membrane, Electroanalysis 12 (2000) 1286-1292.

[8] J. Bobacka, Conducting polymer-based solid-state ion-selective electrodes, Electroanalysis 18 (2006) 7-18.

[9] J. Sutter, A. Radu, S. Peper, E. Bakker, E. Pretsch, Solid-contact polymeric membrane electrodes with detection limits in the subnanomolar range, Anal. Chim. Acta 523 (2004) 53-59.

[10] K.Y. Chumbimuni-Torres, N. Rubinova, A. Radu, L.T. Kubota, E. Bakker, Solid contact potentiometric sensors for trace level measurements, Anal. Chem. 78 (2006) 1318-1322.

[11] J. Sutter, E. Lindner, R.E. Gyurcsányi, E. Pretsch, A polypyrrole-based solidcontact $\mathrm{Pb}^{2+}$-selective PVC-membrane electrode with a nanomolar detection limit, Anal. Bioanal. Chem. 380 (2004) 7-14.

[12] J. Bobacka, M. McCarrick, A. Lewenstam, A. Ivaska, All solid-state poly (vinyl chloride) membrane ion-selective electrodes with poly (3-octylthiophene) solid internal contact, Analyst 119 (1994) 1985-1991.

[13] J. Bobacka, T. Lindfors, M. McCarrick, A. Ivaska, Single-piece all-solid-state ionselective electrode, Anal. Chem. 67 (1995) 3819-3823.

[14] J. Bobacka, T. Lahtinen, J. Nordman, S. Häggström, K. Rissanen, A. Lewenstam, A. Ivaska, All-solid-state $\mathrm{Ag}^{+}$-ISE based on [222] p,p,p-cyclophane, Electroanalysis 13 (2001) 723-726.

[15] J. Wang, Nanomaterial-based electrochemical biosensors, Analyst 130 (2005) 421-426.

[16] A. Düzgün, G.A. Zelada-Guillén, G.A. Crespo, S. Macho, J. Riu, F.X. Rius, Nanostructured materials in potentiometry, Anal. Bioanal. Chem. 399 (2011) $171-181$.

[17] T.J. Yin, W. Qin, Applications of nanomaterials in potentiometric sensors, Trends Anal. Chem. 51 (2013) 79-86.

[18] M. Fouskaki, N. Chaniotakis, Fullerene-based electrochemical buffer layer for ion-selective electrodes, Analyst 133 (2008) 1072-1075.

[19] J.F. Ping, Y.X. Wang, J. Wu, Y.B. Ying, Development of an all-solid-state potassium ion-selective electrode using graphene as the solid-contact transducer, Electroanal. Chem. 13 (2011) 1526-1532.

[20] R. Hernández, J. Riu, J. Bobacka, C. Vallés, P. Jiménez, A.M. Benito, W.K. Maser, F.X. Rius, Reduced graphene oxide films as solid transducers in potentio- metric all-solid-state ion-selective electrodes, J. Phys. Chem. C 116 (2012) 22570-22578.

[21] G.A. Crespo, S. Macho, F.X. Rius, Ion-selective electrodes using carbon nanotubes as ion-to-electron transducers, Anal. Chem, 80 (2008) 1316-1322.

[22] G.A. Crespo, S. Macho, J. Bobacka, F.X. Rius, Transduction mechanism of carbon nanotubes in solid-contact ion-selective electrodes, Anal. Chem. 81 (2009) $676-681$.

[23] J.W. Zhu, Y. Qin, Y.H. Zhang, Preparation of all solid-state potentiometric ion sensors with polymer-CNT composites, Electrochem. Commun. 11 (2009) 1684-1687.

[24] C.Z. Lai, M.A. Fierke, A. Stein, P. Bühlmann, Ion-selective electrodes with threedimensionally ordered macroporous carbon as the solid contact, Anal. Chem. 79 (2007) 4621-4626.

[25] J.B. Hu, X.U. Zou, A. Stein, P. Bühlmann, Ion-selective electrodes with colloidimprinted mesoporous carbon as solid contact, Anal. Chem. 86 (2014) $7111-7118$.

[26] D. Wei, A. Ivaska, Applications of ionic liquids in electrochemical sensors, Anal Chim. Acta 607 (2008) 126-135.

[27] D.V. Chernyshov, V.M. Egorov, N.V. Shvedene, I.V. Pletnev, Low-melting ionic solids: versatile materials for ion-sensing devices, ACS Appl. Mater. Interfaces 1 (2009) 2055-2059.

[28] N. Maleki, A. Safavi, F. Tajabadi, High-performance carbon composite electrode based on an ionic liquid as a binder, Anal. Chem. 78 (2006) 3820-3826.

[29] A. Safavi, N. Maleki, F. Honarasa, F. Tajabadi, F. Sedaghatpour, Ionic liquids modify the performance of carbon based potentiometric sensors, Electroanalysis 19 (2007) 582-586.

[30] E. Bakker, Determination of improved selectivity coefficients of polymer membrane ion-selective electrodes by conditioning with a discriminated ion, J. Electrochem. Soc. 143 (1996) L83-L85.

[31] S. Kamaata, A. Bhale, Y. Fukunaga, H. Murata, Copper(II)-selective electrode using thiuram disulfide neutral carriers, Anal. Chem. 60 (1988) 2464-2467.

[32] J.F. Ping, Y.X. Wang, Y.B. Ying, J. Wu, Application of electrochemically reduced graphene oxide on screen-printed ion-selective electrode, Anal. Chem. 84 (2013) 3473-3479.

[33] S.Y. Yu, F.H. Li, W. Qin, An all-solid-state $\mathrm{Cd}^{2+}$-selective electrode with a low detection limit, Sens. Actuators B 155 (2011) 919-922.

[34] W. Qin, R.N. Liang, X.L. Fu, Q.W. Wang, T.J. Yin, W.J. Song, Trace-level potentiometric detection in the presence of a high electrolyte background Anal. Chem. 84 (2012) 10509-10513.

[35] M. Fibbioli, K. Bandyopadhyay, S.G. Liu, L. Echegoyen, O. Enger, F. Diederich, D. Gingery, P. Bühlmann, H. Persson, U.W. Suter, E. Pretsch, Redox-active selfassembled monolayers for solid-contact polymeric membrane ion-selective electrodes, Chem. Mater. 14 (2002) 1721-1729.

[36] X.U. Zou, J.H. Cheong, B.J. Taitt, P. Bühlmann, Solid contact ion-selective electrodes with a well-controlled $\mathrm{Co}(\mathrm{II}) / \mathrm{Co}(\mathrm{III})$ redox buffer layer, Anal. Chem. 85 (2013) 9350-9355.

[37] J. Bobacka, A. Lewenstam, A. Ivaska, Electrochemical impedance spectroscopy of oxidized poly(3,4-ethylenedioxythiophene) film electrodes in aqueous solutions, J. Electroanal. Chem. 489 (2000) 17-27.

[38] J.P. Veder, R. De Marco, G. Clarke, R. Chester, A. Nelson, K. Prince, E. Pretsch, E. Bakker, Elimination of undesirable water layers in solid-contact polymeric ion-selective electrodes, Anal. Chem. 80 (2008) 6731-6740. 